\title{
Methodological validation of measuring Hanwoo hair cortisol concentration using bead beater and surgical scissors
}

\author{
Jalil Ghassemi Nejad ${ }^{1,2}$, Mohammad Ataallahi ${ }^{1}$ and Kyu-Hyun Park ${ }^{1 *}$ \\ ${ }^{1}$ College of Animal Life Sciences, Kangwon National University, Chuncheon 24341, Korea \\ ${ }^{2}$ Team of An Educational Program for Specialists in Global Animal Science, Brain Korea 21 Plus Project, Konkuk University, Seoul 05029, \\ Korea
}

\begin{abstract}
Different methodologies in hair cortisol extraction may alter the final output. Thus finding the standard methodology according to a laboratory facilities is pivotal. This study was carried out to validate the feasibility of two methods of grinding hair for cortisol extraction in Korean native (Hanwoo) cattle. Hair from nine cattle including mature cows, heifers, and calves were assigned to one of the following methods for grinding hair; 1) using bead beater (BB) and 2) using surgical scissors (SS). Hair samples (> $1 \mathrm{~g}$ ) were harvested from forehead of each individual twice (first and second measurement) to validate the results. To improve the accuracy of the obtained data, each sample was duplicated into two wells during enzyme immunoassay (EIA) analysis. Overall comparison of hair cortisol concentration (HCC) showed that the data within the range (out of the range) of standards provided by the EIA kit were $88.9 \%(11.1 \%)$ and $66.7 \%(33.3 \%)$ for BB compared with SS, respectively. In the first measurement, application of BB was tended to show higher $(p=0.056)$ amount of HCC compared with SS. In the second measurement application of BB showed higher $(p=0.0028)$ amount of HCC compared with SS. Among the cattle, calves showed higher HCC using BB compared with SS $(p<0.05)$. Application of BB in hair grinding methodology for Hanwoo cattle may improve cortisol extraction in comparison to application of SS method, with more consistency. Thus, it would be the preferable method to use.
\end{abstract}

Keywords: Bead beater, Hair cortisol methodology, Hanwoo cattle, Surgical scissors

\section{Introduction}

Blood, saliva, urine, and feces as common different biological matrices have been routinely applied to measure acute stress levels in animals and humans $[1,2]$. However, these biological matrices require specific storage (i.e. refrigerator, freezer, etc.) before the analysis and also the measurements are vulnerable to parameters such as time of collection, animal restrain stress, etc [2]. Furthermore, the aforementioned matrices need repeated measurements which are costly, and the collections of samples are invasive [2,3]. A previous study has shown the presence of cortisol in hair of cattle as a putative biomarker of chronic stress [4]. Hence, the hair of animals has been employed for the measurement since the sample collection was non-invasive, it could be stored at room temperature, and also the data indicated prolonged stress exposure without repeating the measurements [2]. However, a lack of studies in validation of

Received: Nov 22, 2018 Revised:Jan 7, 2019 Accepted:Jan 14,2019

"Corresponding author: Kyu-Hyun Park, College of Animal Life Sciences, Kangwon National University, Chuncheon 24341, Korea.

Tel: +82-33-250-8621, E-mail: kpark74@kangwon.ac.kr

This is an Open Access article distributed under the terms of the Creative Commons Attribution Non-Commercial License (http://creativecommons.org/licenses/by$\mathrm{nc} / 4.0 /$ ) which permits unrestricted non-commercial use, distribution, and reproduction in any medium, provided the original work is properly cited.

Copyright (C) 2019 Korean Society of Animal Science and Technology. 


\section{JAST}

hair cortisol extraction methodologies were observed that these methodologies could be sources of variations in determination of hair cortisol prior to cortisol extraction. Likewise, previous literatures indicated three main methods to grind or finely cut the hair into small pieces for cortisol extraction including a) using surgical scissors (SS) [5, 6]; b) bead beater (BB) [7, 8], and c) ball mill [8, 9]. Each of these methods has claimed to have limitations. The main differences between these methods included particle size [10], mass homogenization, time economy [11], hair nature, laborious and cost-effectiveness, availability of the required facilities in laboratories, and being hygienic that could possibly confound the amount of cortisol extraction [5, 8]. For instance, application of ball mill was reported to be costly where suited for grinding of only two samples at once [8]. In order to extract cortisol from the hair shaft, the follicles should have been broken down to the smallest particles as possible, for maximum accuracy. In addition, it has been noted that the more efficient cortisol extraction from the hair shaft revealed better accuracy of methodology and reported to be more reliable [10]. In this regard, this study was designed to validate two main grinding methods of Hanwoo cattle' hair for cortisol extraction including $\mathrm{BB}$ and $\mathrm{SS}$.

Unlike SS, BB reduced contamination and was indicated as a less time consuming method when multiple samples were processed. Moreover, studies that used SS have reported overall smaller concentrations of cortisol $[10,12]$ except for the study conducted by Slominski et al. [13] that found similar level of cortisol extracted by milling and finely cutting hair. Collectively, a lack of literature, particularly in Hanwoo cattle in regard to methodological validation of hair cortisol extraction was the motivation of the current study.

\section{Methods}

\section{Animals and hair sampling site}

The cattle used in the current study were kept in the university farm under the same environmental conditions and were fed with regular diet procedure of the farm where fresh water was always available. Nine healthy Korean (Hanwoo) cattle, including mature cows, heifers, and calves ( $\mathrm{n}=3$ each $\times$ two duplications $=6$ per type of cattle), with initial average body weight $(\mathrm{kg})=545 \pm 93.9,380.7$ \pm 68.5 , and $53.7 \pm 1.4$, and average age (month) $=55.9 \pm 13.6,17.2$ $\pm 0.3,1.8 \pm 0.6$, respectively, were employed prior to the beginning of the experiment. Hair samples from the forehead of each individual were harvested twice in the first and second measurements to repeat the measurement in order to improve the reliability of the assessment.

\section{Hair sample preparation procedure}

The procedure of hair cortisol extraction was carried out in accor- dance with Davenport et al. [14] and Ghassemi Nejad et al. [5, $15]$ in the current study. In short, preparing of hair cortisol analysis included shaving from the forehead of each individual, wrapping in aluminum foil, unwrapping and washing with propanol, and grinding into powder, application of methanol, drying, and then applying HCC assay using the Enzyme Immunoassay (EIA) kit (Salimetrics, State College, USA) according to the manufacturer's recommendation. In detail, hair samples ( $>1 \mathrm{~g}$ ) were shaved carefully from cow's forehead $(10-20 \mathrm{~cm})$, closest to the skin as much as possible using electric hair shaver (Hair Clippers, Model 7200, RIKEI ${ }^{\circledR}$ Korea). Afterward, the hair samples were immediately folded with sheets of aluminum foil, numbered, placed into dry polypropylene tubes (50 mL Conical Tube, HM Hyundai Micro Co., Korea), stored in a plastic bag, transported to the laboratory, and stored at room temperature for the following procedures including washing, cortisol extraction, drying and analyzing by EIA (Fig. 1).

\section{Washing hair samples}

Aluminum foils inclusion hair samples were unwrapped and then, weighed $250 \mathrm{mg}$ from each sample using digital scale (Mettler Toledo, ME204). Afterwards the hair samples were transferred into new polypropylene tubes $(15 \mathrm{~mL}$ Conical Tube, HM Hyundai Micro Co., Korea). Following that $5 \mathrm{~mL}$ of propanol (Daejung Chemicals \& Metals CO., Gyeonggi-do, Korea) was added to each tube by a single channel pipette $\left(5000 \mu \mathrm{L}, \mathrm{VITLAB}^{\circledR}{ }^{\circledR}\right.$ Grossostheim, Germany). The tubes were then shaken gently for $3 \mathrm{~min}$ to wash external cortisol. This procedure was repeated twice followed by drying hair samples for 7 days at room temperature.

\section{Grinding hair samples}

Hair pulverization was performed by two accessible instruments in our laboratory; BB (tacoTMPrep, 50/60 Hz 2A, GeneReach Biotechnology Corp, Taichung City, Taiwan) and SS. Fifty mg portions of hair samples were transferred into tubes of $\mathrm{BB}(2 \mathrm{~mL})$ containing six stainless steel ball and were ground into powder for $8 \mathrm{~min}$ at $50 \mathrm{~Hz}$. Fifty $\mathrm{mg}$ of the same hair samples were finely cut (> $1-2 \mathrm{~mm}$ ) using sterilized SS and put into the micro tubes $(1.5 \mathrm{~mL})$.

\section{Cortisol extraction}

One $\mathrm{mL}$ of methanol (Daejung Chemicals \& Metals CO., Gyeonggi-do, Korea) delivered into the tubes contained hair powder by a pipette (Research Plus ${ }^{\circledR}$ Eppendorf pipette). A while later, samples were put on a tube rotator (Multi Mixer, SLRM-3 Mylab, Seoulin Bioscience, Korea) in slow rotation $(0.026 \times \mathrm{g})$, for 24 hours at room temperature to extract cortisol. Afterwards, the samples were centrifuged (Labogene ${ }^{\circledR} 1730 \mathrm{R}$, Korea) at $14,269 \times \mathrm{g}$ for 60 seconds at room temperature. Following centrifugation $0.6 \mathrm{~mL}$ of supernatant was pipetted (Research Plus ${ }^{\circledR}$ Eppendorf pipette) 


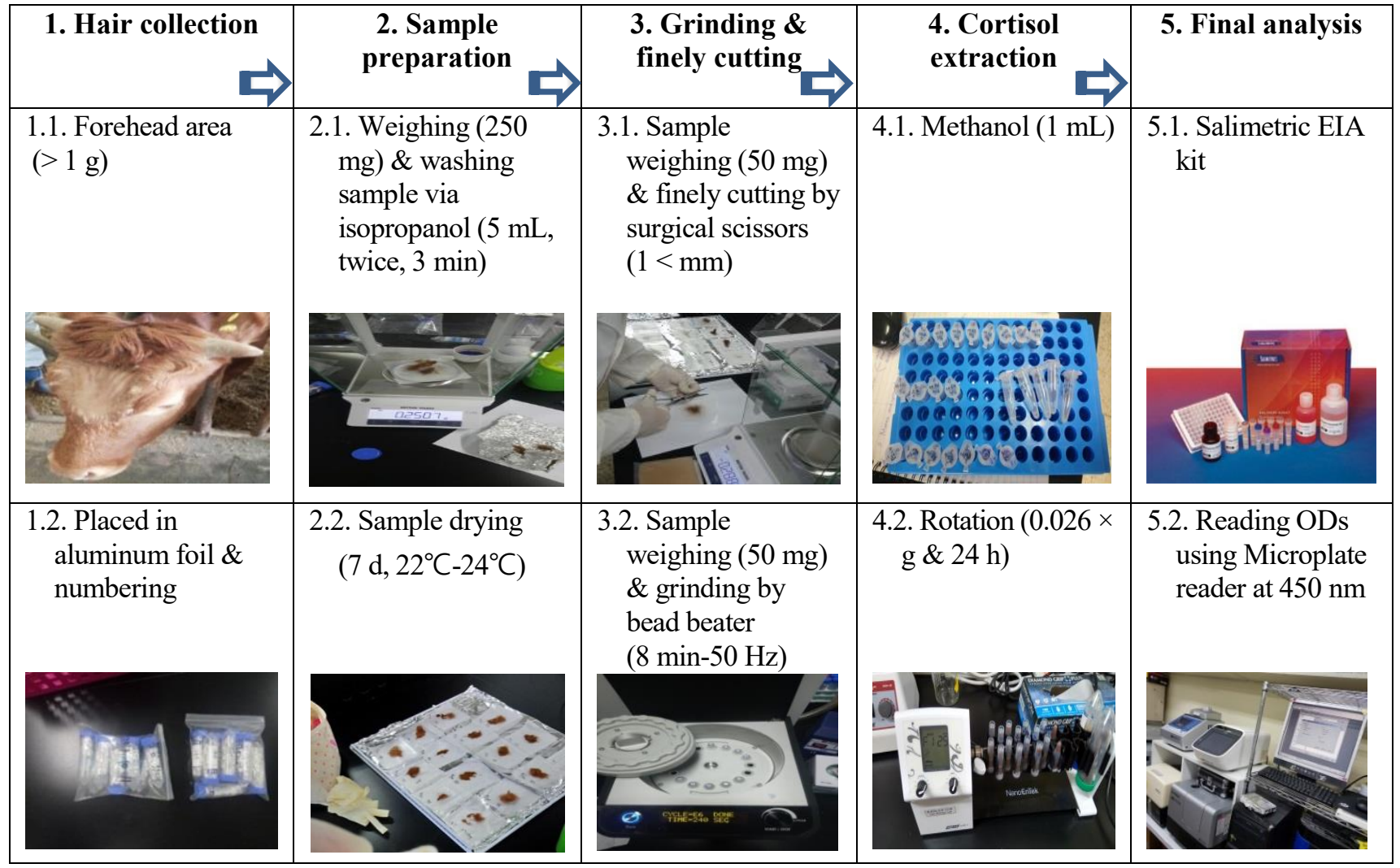

Fig. 1. Info-graphical abstract of hair cortisol analysis methodology.

and transferred into new microcentrifuge tube $(1.5 \mathrm{~mL})$ and incubated at incubator (Sanyo, JP/MCO175) for drying at $38^{\circ} \mathrm{C}$ in order to evaporate the methanol. The dried hair samples were thawed at room temperature prior to use the EIA kit. Following by adding $400 \mu \mathrm{L}$ of phosphate buffer (EIA kit), then mixed completely with a vortex (VTX-3000L, LMS Co. LTD, Tokyo, Japan), and were centrifuged at $1,500 \times \mathrm{g}$ for 15 minutes. And then each sample was run in duplicate in order to improve the power of the test and reliability of the results. For duplication, $25 \mu \mathrm{L}$ of supernatant have chosen based on the kit recommendations. It should be noted that 6 standard solutions were used to draw the data curve and the results over/lower the range of standards were considered as outliers and were consequently omitted. The microplate reader (Synergy ${ }^{\mathrm{TM}}$ H1, BioTek, USA) was used to read the optical density of samples at a wavelength of $450 \mathrm{~nm}$. Afterwards, a free 4 parameter non linear regression curve fit (MyAssay Analysis Online Software

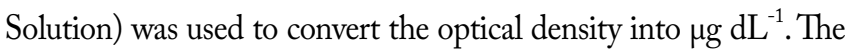
conversion of the obtain data from the software calculated into pg $\mathrm{mg}^{-1}$ using the following equation:

$$
(\mathrm{A} / \mathrm{B}) \times(\mathrm{C} / \mathrm{D}) \times \mathrm{E} \times 10,000=\mathrm{F}
$$

where $\mathrm{A}=\mu \mathrm{g} \mathrm{dL}^{-1}$ from assay output; $\mathrm{B}=$ weight $(\mathrm{mg})$ of hair sub- jected to extraction; $\mathrm{C}=$ volume $(\mathrm{mL})$ of methanol added to the powdered hair; $\mathrm{D}=$ volume $(\mathrm{mL})$ of methanol recovered from the extract and subsequently dried down; $\mathrm{E}=$ volume $(\mathrm{mL})$ of assay buffer used to reconstitute the dried extract; and $\mathrm{F}=$ final value of HCC (pg mg $\left.{ }^{-1}\right)$ [8].

\section{Statistics}

Calculation of data was performed using mixed procedure of SAS software (Version 9.3, SAS Institute Inc., NC., USA 2010) in order to compare two grinding methods BB and SS. Differences between means were tested by Tukey and then determined with $p$-value. The mean HCC at first analysis was compared with the mean HCC of second analysis. The estimates and standard errors provided in this work were calculated from the least square means of the fixed effects in the model. Statistical differences were considered significant at $p<0.05$ and differences among means with 0.05 $<p<0.10$ was accepted as representing tendencies to differences.

\section{Results and Discussion}

Hair cortisol concentrations (HCC) were considered within the range of standards $\left(0.012\right.$ to $\left.3.000 \mu \mathrm{g} \mathrm{dL}^{-1}\right)$ provided by the EIA kit. Thus, any obtained data out of these ranges were considered 
outlier . Given the range of six standard solutions overall comparison of HCC showed that the data within the range of standards (out of the range of standards) were $88.9 \%$ (11.1\%) and $66.7 \%$ (33.3\%) from duplicated HCC in the first and second measurements (Fig. 2) for BB compared to SS, respectively. Extraction of hair cortisol indicated to be dependable on the hair particle size and therefore the higher the amount of powder the higher the concentration of extracted cortisol would be $[10,12]$. Accordingly, based on two different grinding methods that were used in the present study we depicted that using BB was relatively easier to perform and more portion of cortisol could be extracted as compared with SS. In addition, application of $\mathrm{BB}$ showed higher capability to grind more samples (16-24) at a time compared with SS. Also, using BB provided homogenized ground hair which increased the chance of higher potion of cortisol extraction and thus improved the reliability of the assessment. The greater amount of obtained cortisol by $\mathrm{BB}$ may be related to the homogenized and smaller particles of hair compared with SS. In agreement with the present study, it was suggested that milled samples may yield up to 3.5 times cortisol compared with application of SS and less variation among the obtained data $[10,15]$. The application of SS in finely cutting hair was reported in several studies $[5,14,15]$. Additionally, in this experiment we observed that using SS was shown not to be an applicable tool when the size of hair was too small. It should be noted that the ground hair obtained from SS was not homogenized that may confound the reliability of the assessment. Using SS was time consuming and possible contaminations might be increased. More importantly application of SS increased outliers data of cortisol extraction compared with BB as we observed in the current study. Given these results application of BB for grinding hair instead of SS could be suggested.

The results indicated that HCC showed a tendency of being significantly higher $(p=0.056)$ in $\mathrm{BB}$ compared with SS in the first measurement (Fig. 3A). The necessity of selecting a precise method

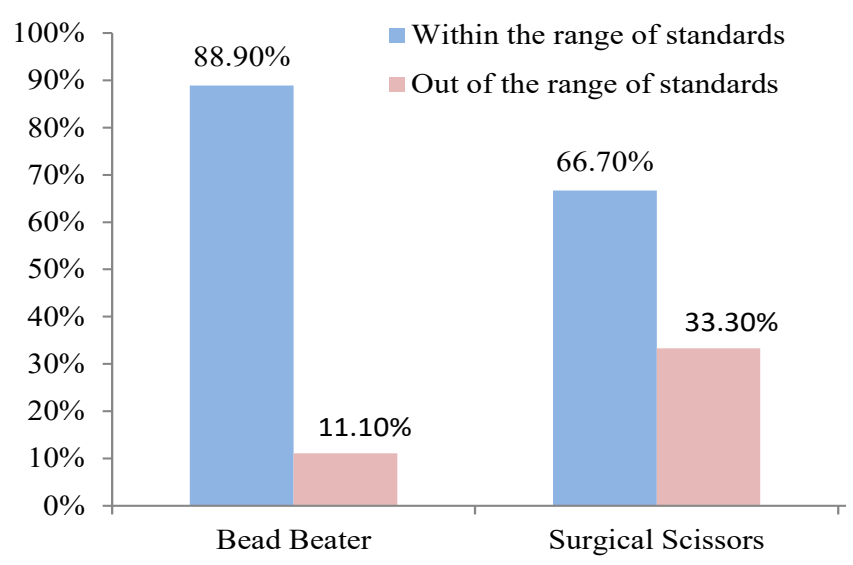

Fig. 2. Comparison of grinding methods within the range (out of the range) of standards provided by the enzyme immunoassay kit in the first and the second measurement. to detect hair cortisol hormone as a putative biomarker of chronic stress seemed crucial. In order to increase the portion of cortisol extraction from the hair shaft, the hair samples need to be ground into a fine powder to break down the hair's protein matrix and increasing the surface area for cortisol extraction [8]. The length of hair could be finely cut to approximately $1 \mathrm{~mm}$ with $\mathrm{SS}$ whereas $\mathrm{BB}$ produced hair with finest powder.

Highly significant difference $(p=0.0028)$ was observed in HCC using $\mathrm{BB}$ compared with $\mathrm{SS}$ in the second measurement (Fig. 3B). A better extraction yield as a result of the more homogenous and finely ground hair matrix could explain the differences. In the second measurement, the increase in HCC was likely caused by the increased surface area created by grinding the hair into much powder using BB from what we normally expected by SS. The result of the second measurement was consistent with another study on hair of human and monkey that reported higher amount of cortisol extraction using BB compared with SS [8] and in monkey [14] using ball mill compared with SS. In spite of the present study, another

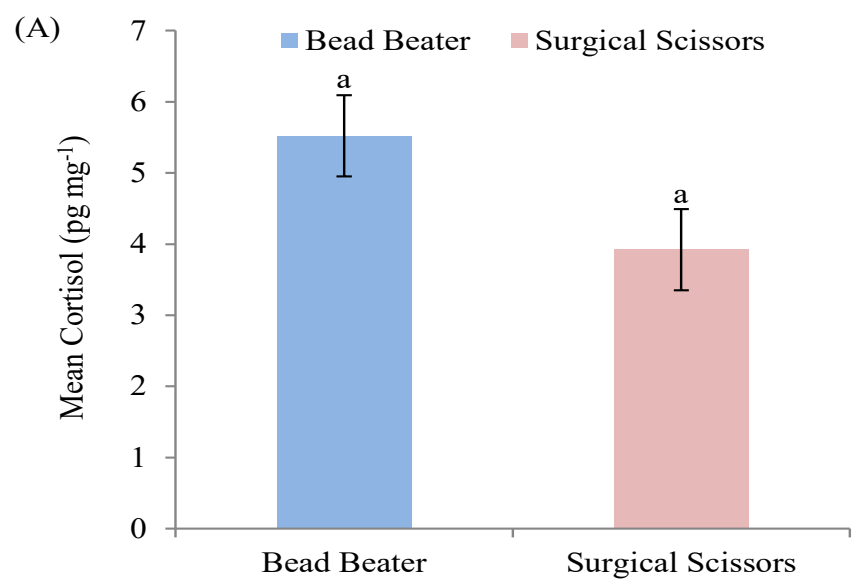

First measurement

(B)

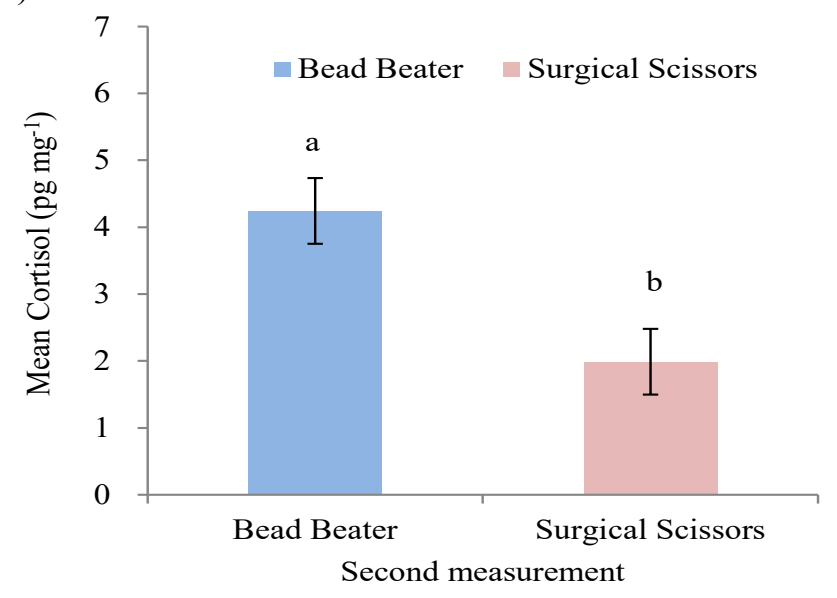

Fig. 3. Effect of two grinding methods on HCC in Hanwoo cattle (mean \pm SEM). Different letters $(a, b)$ represent significant differences $(p<0.05)$ in the first $(A)$, and the second $(B)$ measurement. 
report in human showed that milling hair using Bullet Blender (Next Advance Inc., Averill Park, USA) did not extract more cortisol than finely cutting by SS [13]. They concluded that using SS for finely cutting hair was more effective procedure than using mill method in HCC measurement.

Calves showed higher $(p<0.05)$ HCC using BB compared with SS in the first (Fig. 4A) and second measurement (Fig. 4B). However, no significant differences were observed in HCC among the mature cows and heifers for the both measurements in BB compared with SS (Fig. 4A, 4B). Additionally, higher HCC in calves using $\mathrm{BB}$ compared with $\mathrm{SS}$ could be attributed to the fact that calves were more vulnerable due to being newborn and habituation to the new life environment than other cattle. This phenomenon has also previously been reported by Comin et al. [12]

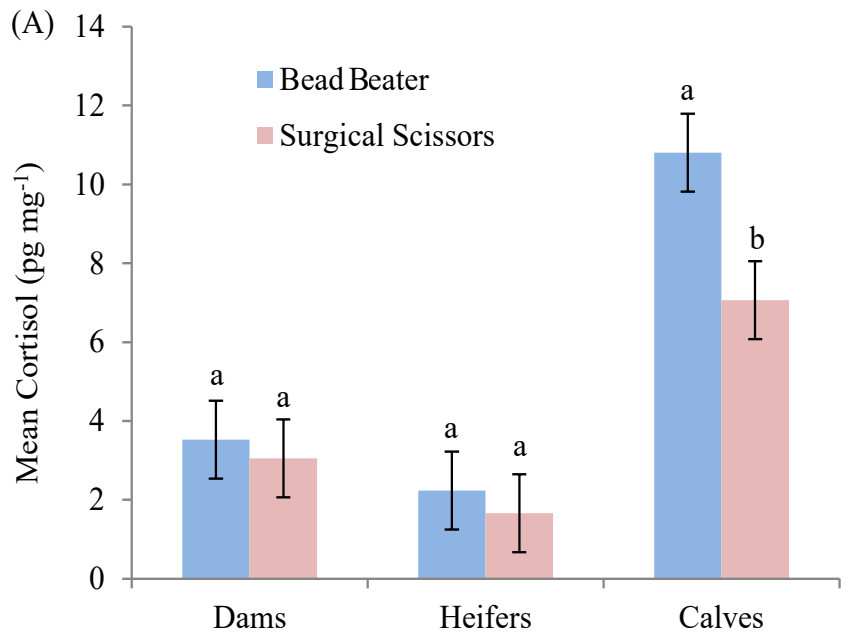

First measurement

(B)

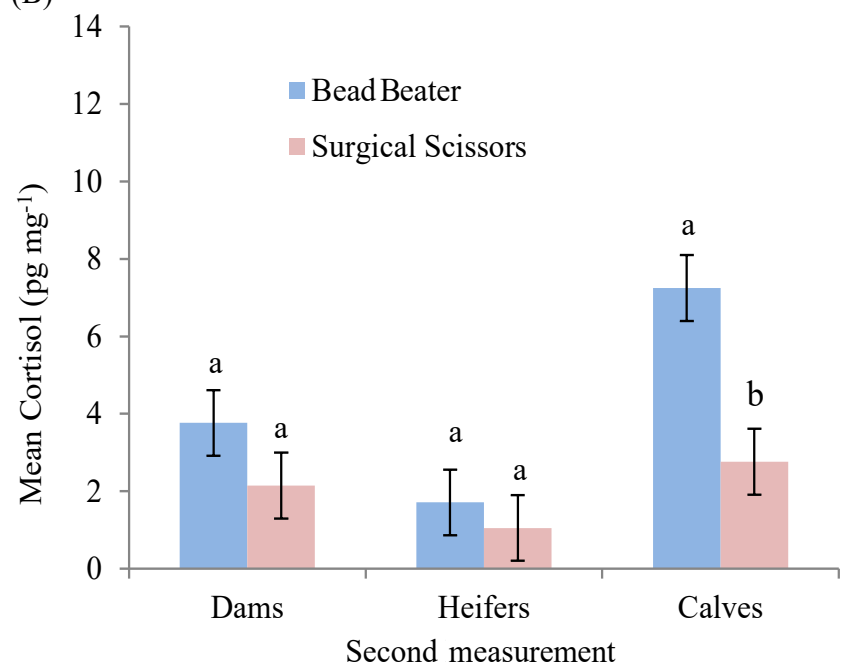

Fig. 4. Effect of two grinding methods on HCC among Hanwoo cattle (mean \pm SEM). Different letters $(a, b)$ represent significant differences $(p<$ $0.05)$ in the first $(A)$, and the second $(B)$ measurement. who reported higher amount of cortisol in Holstein young calves than lactating cattle. This could be possibility a reason why calves showed higher HCC in both measurements.

\section{Conclusion}

Inability for finely cutting hair by SS resulted in inconsistent data, lower amount of HCC, and thus inappropriate methodology particularly in small hair samples. The use of BB as an automated homogenizer resulted in finely powder and homogenized hair particles that maximized the amount of cortisol extraction. Therefore, grinding hair samples by $\mathrm{BB}$ was preferable methodology in Hanwoo hair cortisol extraction compared with SS.

\section{Competing interests}

No potential conflict of interest relevant to this article was reported.

\section{Funding sources}

This work was carried out with the support of "Cooperative Research Program for Agriculture Science \& Technology Development (Project title: Livestock productivity change analysis with climate change, Project No. PJ012771)" Rural Development Administration, Republic of Korea and of 2017 research Grant from Kangwon National University.

\section{Acknowledgements}

Not applicable.

\section{Availability of data and materials}

Upon reasonable request, the datasets of this study can be available from the corresponding author.

\section{Authors'contributions}

Ghasseminejad J and Park KH have defined conceptualization and data curation. Ghasseminejad J has suggested the methodology, validation, and has written the original draft. Ataallahi $M$ has performed software and formal analysis. All authors investigated the research. Park KH supported the project financially, reviewed and edited the final manuscript.

\section{Ethics approval and consent to participate}

This article does not require IRB/IACUC approval because there are no human and animal participants.

\section{ORCID}

Jalil Ghassemi Nejad

https://orcid.org/0000-0001-6578-8829

Mohammad Ataallahi

https://orcid.org/0000-0003-0234-8863

Kyu-Hyun Park 


\section{JAST}

\section{References}

1. Nejad JG, Jeong C, Shahsavarani H, Sung KIL, Lee J. Embedded dental cortisol content: a pilot study. Endocrinol Metab Syndr. 2016;5:240.

2. Russell E, Koren G, Rieder M, Van Uum S. Hair cortisol as a biological marker of chronic stress: current status, future directions and unanswered questions. Psychoneuroendocrinology. 2012;37:589-601.

3. Stalder T, Kirschbaum C. Analysis of cortisol in hairstate of the art and future directions. Brain Behav Immun. 2012;26:1019-29.

4. Gonzalez-de-la-Vara Mdel R, Valdez RA, Lemus-Ramirez V, Vazquez-Chagoyan JC, Villa-Godoy A, Romano MC. Effects of adrenocrticotropic hormone challenge and age on hair cortisol concentrations in dairy cattle. Can J Vet Res. 2011;75:216-21.

5. Ghassemi Nejad J, Lohakare JD, Son JK, Kwon EG, West JW, Sung KI. Wool cortisol is a better indicator of stress than blood cortisol in ewes exposed to heat stress and water restriction. Animal. 2014;8:128-32.

6. Accorsi PA, Carloni E, Valsecchi P, Viggiani R, Gamberoni M, Tamanini C, Seren E. Cortisol determination in hair and faeces from domestic cats and dogs. Gen Comp Endocrinol. 2008;15:398-402.

7. Hodes A, Lodish MB, Tirosh A, Meyer J, Belyavaskaya E, Lyssikatos $\mathrm{C}$, et al. Hair cortisol in the evaluation of cushing syndrome. Endocrine. 2017;56:164-74.

8. Meyer J, Novak M, Hamel A, Rosenberg K. Extraction and analysis of cortisol from human and monkey hair. J Vis Exp. 2014;83:e50882.

9. Moya D, Schwartzkopf-Genswein KS, Veira DM. Standardization of a non-invasive methodology to measure cortisol in hair of beef cattle. Livest Sci. 2013;158:138-44.

10. Burnett TA, Madureira AML, Silper BF, Nadalin A, Tahmasbi A, Veira DM, et al. Short communication: factors affecting hair cortisol concentrations in lactating dairy cows.J Dairy Sci. 2014;97:7685-90.

11. Gibbons EL, Brangs CGH, Burden WD. Bead beater: aprimer. In: OPS Diagnostics. 2014. https://opsdiagnostics.com/ notes/ranpri/OPSD_Bead_Beating_Primer_2014\%20v1.pdf. Accessed 10 Jan 2019.

12. Comin A, Peric T, Corazzin M, Verones MC, Meloni T, Zufferli V, et al. Hair cortisol as a marker of hypothalamic-pituitary-adrenal axis Activation in Friesian dairy cows clinically or physiologically compromised. Livest Sci. 2013;152:36-41.

13. Slominski R, Rovnaghi CR, Anand KJ. Methodological considerations for hair cortisol measurements in children. Ther Drug Monit. 2015;37:812-20.

14. Davenport MD, Tiefenbacher S, Lutz CK, Novak MA, Meyer JS. Analysis of endogenous cortisol concentrations in the hair of rhesus macaques. Gen Comp Endocrinol. 2006;147:25561.

15. Nejad JG, Kim BW, Lee BH, Kim JY, Sung KI. Effects of water addition to total mixed ration on water intake, nutrient digestibility, wool cortisol and blood indices in corriedale ewes. Asian-Australas J Anim Sci. 2017;30:1435-41. 